\title{
Universal power law tails of time correlation functions
}

\author{
M. H. Ernst \\ CNLS, Los Alamos National Laboratory, Los Alamos, New Mexico 87545, USA and Institute for Theoretical Physics, \\ University of Utrecht, Princetonplein 5, P.O. Box 80.195, 3508 TD Utrecht, The Netherlands
}

(Received 8 November 2004; published 11 March 2005)

\begin{abstract}
The universal power law tails of single particle and multiparticle time correlation functions are derived from a unifying point of view, solely using the hydrodynamic modes of the system. The theory applies to general correlation functions and to systems more general than classical fluids. Moreover, it is argued that the collisional transfer part of the stress-stress correlation function in dense classical fluids has the same long-time tail $\sim t^{-1-d / 2}$ as the velocity autocorrelation function in Lorentz gases.
\end{abstract}

DOI: 10.1103/PhysRevE.71.030101

PACS number(s): 05.20.Dd, 05.40.-a

The long-time tail (LTT) of the velocity autocorrelation function $\left\langle v_{x}(t) v_{x}(0)\right\rangle \sim t^{-d / 2}$ in $d$-dimensional fluids in thermal equilibrium is the prototypical example of time correlation functions in fluids showing universal power-law behavior, independent of the details of the interparticle interactions $[1,2]$. The goal of this paper is to present, from a unified point of view, a quantitative description of the asymptotic decay of single-particle and multiparticle time correlation functions (TCF's). The starting point is the mode coupling theory for classical fluids in [2], which is extended to more general systems, and more general TCF's.

What about the asymptotic decay of other single particle properties that are coupled to a conservation law? Consider the single site $(\mathbf{n}=\mathbf{0})$ correlation function $\left\langle\mathbf{v}_{0}(t) \mathbf{v}_{0}(0)\right\rangle$, initialized in a state of thermal equilibrium, which state may or may not be maintained by the dynamic evolution of the system. The system considered has fixed spins or velocity vectors $\mathbf{v}_{\mathbf{n}}$ at the sites $\mathbf{n}=\left\{n_{1}, n_{2}, \ldots, n_{d}\right\}$ of a $d$-dimensional cubic lattice with nearest neighbor (NN) interactions conserving the total magnetization or momentum, $\Sigma_{\mathbf{n}} \mathbf{v}_{\mathbf{n}}(t)$. For convenience we take periodic boundary conditions. Such models are Glauber's model with Ising spins at zero temperature [3], or lattice gas cellular automata with $b$ velocities per site associated with the links [4], or granular fluid models with a discrete or continuous velocity vector assigned to each site $[5,6]$. In the following we consider as an example an exactly soluble model of a granular fluid on a lattice whose LTT's are also covered by the results, to be derived in this Rapid Communication.

Here a pair of $\mathrm{NN}$ sites interact at a rate $\kappa_{0}$ such that $\mathbf{v}_{\mathbf{n}}$ and $\mathbf{v}_{\mathbf{m}}$ are each replaced by their arithmetic mean. Then the equation of motion for the mean value, $\left\langle\mathbf{v}_{\mathbf{n}}\right\rangle \equiv \mathbf{V}_{\mathbf{n}}$, can be written in appropriate units of length and time as a discrete diffusion equation, i.e.,

$$
d \mathbf{V}_{\mathbf{n}} / d \tau=\frac{1}{2} \sum_{\mathbf{a}}\left[\mathbf{V}_{\mathbf{n}+\mathbf{a}}-\mathbf{V}_{\mathbf{n}}\right]=\frac{1}{2} \Delta \mathbf{V}_{\mathbf{n}}
$$

Here $\tau=2 D t / a^{2}$ is the rescaled time, $D=\kappa_{0} a^{2}$ the diffusion coefficient, $a$ the lattice distance, a runs over NN sites, and $\Delta$ is the Laplace operator on a $d$-dimensional cubic lattice. The exact solution $[3,6]$ of this equation is $\mathbf{V}_{\mathbf{n}}(\tau)$ $=\sum_{\mathbf{m}} \mathbf{V}_{\mathbf{n}-\mathbf{m}}(0) \Pi_{i=1}^{d}\left[I_{m_{i}}(\tau) e^{-\tau}\right]$, where $I_{m}(\tau)$ is the modified
Bessel function. The space-time correlation function $\left\langle v_{\mathbf{n} x}(t) v_{\mathbf{0} x}(0)\right\rangle$ satisfies the same equation as $\left\langle v_{\mathbf{n} x}\right\rangle(t)$, where $\langle\cdots\rangle$ is an ensemble average over an arbitrary initial state. In the sequel all TCF's refer to initial states in thermal equilibrium. They are not necessarily spatially uniform. The single site TCF, $C_{0}(t)=\left\langle v_{\mathbf{0} x}(t) v_{\mathbf{0} x}(0)\right\rangle /\left\langle v_{\mathbf{0} x}^{2}\right\rangle$, follows by setting $\mathbf{n}$ $=\mathbf{0}$, and yields the exact solution

$$
C_{0}(t)=\left[e^{-\tau} I_{0}(\tau)\right]^{d} \sim[2 \pi \tau]^{-d / 2},
$$

where the asymptotic equality gives its LTT. Here $C_{0}(t)$ is essentially the return probability of initial momentum to its points of origin. The same exact results apply to the spin TCF in the Glauber model at zero temperature, and to the energy autocorrelation function $C_{e}(t)$ $=\left\langle\delta \epsilon_{\mathbf{n}}(t) \delta \epsilon_{\mathbf{n}}(o)\right\rangle /\left\langle(\delta \epsilon)^{2}\right\rangle$ in case the total energy $\Sigma_{i} \epsilon_{i}(t)$ is conserved, where $\delta \epsilon_{\mathrm{n}}=\epsilon_{\mathrm{n}}-\langle\epsilon\rangle$. This function is again a return probability.

In all previous models particles have only NN interactions, and are placed on lattice sites. These restrictions will be removed. When the particles are moving, as in fluids, one may also consider the tagged particle fluctuations, $j_{2}=v^{2}-\left\langle v^{2}\right\rangle$, or more generally $j_{2 k}=v^{2 k}-\left\langle v^{2 k}\right\rangle$, and $j_{2 k+1}$ $=v^{2 k} v_{x}$, with $k=0,1, \ldots$, and even $j_{0}=\delta \epsilon$ in case the tagged particle has an additional internal degree of freedom with a discrete or continuous energy $\epsilon$. Here the total energy of the system is conserved, and we are interested in the LTT of these TCF's. Such systems are classical fluids [1], DPD fluids (dissipative particle dynamics), which are mesoscopic models $[7,8]$ with particle, momentum, and possibly energy conservation, or the LBE (Lattice-Boltzmann equation) method [9], which lacks energy conservation. The DPD fluid and the LBE method can be considered as preaveraged versions of, respectively, the Liouville equation and the dynamic equations of lattice gas cellular automata [4], where the rapid short-range fluctuations have been averaged out.

In the DPD fluids the evolution equations for $\left\{\epsilon_{i}(t), \mathbf{v}_{i}(t)\right\}$ are formulated as coupled Langevin equations with dissipative and stochastic pair-interaction terms, having a finite interaction range $r_{c}$, and satisfying the fluctuation dissipation theorem. The stochastic interactions contain, in general, multiplicative white noise [10]. One may also quench the translational degrees of freedom, and freeze the particles at fixed 
random positions, or in fixed periodic lattice configurations. In the former case a final average over quenched configurations has to be performed. Here we consider only the quenched DPD fluid with internal energy states, referred to as a DPD solid $[10,11]$, where dissipative and random interactions conserve the total internal energy. This quenched model of "interacting heat particles" is in several respects the dual model of the overlapping Lorentz gas [10].

The purpose of this paper is to study the LTT's of singleparticle and multiparticle TFC's, $\left\langle J_{b}(t) J_{b}(0)\right\rangle$, in different models, and away from percolation and other critical points, using mode coupling theory. We start with fluid models. Here the LTT's can be concisely summarized in the KadanoffSwift formula [2]

$$
\left\langle J_{b}(t) J_{b}(0)\right\rangle \simeq \frac{1}{2} \int_{\mathbf{k}} \sum_{\lambda \mu}\left[A_{b}^{\lambda \mu}\right]^{2} e^{\left(z_{k}^{\lambda}+z_{k}^{\mu}\right) t},
$$

where $\int_{\mathbf{k}}=V^{-1} \Sigma_{\mathbf{k}}$. In the thermodynamic limit $\int_{\mathbf{k}}$ is replaced by $(2 \pi)^{-d} \int d \mathbf{k}$. The $\lambda \mu$ sum extends over the complete set of hydrodynamic modes $\left\{a_{\mathbf{k}}^{\lambda}\right\}$ of the system. The amplitude $A_{b}^{\lambda \mu}=\left(J_{b} \mid a_{\mathbf{k}}^{\lambda} a_{-\mathbf{k}}^{\mu}\right) \equiv V^{-1}\left\langle J_{b} a_{\mathbf{k}}^{\lambda} a_{-\mathbf{k}}^{\mu}\right\rangle$ represents the component of $J_{b}$ parallel to a product of two modes. These modes are linear combinations of Fourier transforms of conserved densities, and are normalized as $\left(a_{\mathbf{k}}^{\lambda} \mid a_{\mathbf{k}}^{\mu}\right)=\delta_{\lambda \mu}$. The scalar products are defined as $\left(A_{\mathbf{k}} \mid B_{\mathbf{k}}\right)=V^{-1}\left\langle A_{\mathbf{k}} B_{-\mathbf{k}}\right\rangle$, averaged over an equilibrium ensemble. All TCF's considered here approach zero for large times, which implies that $\left(J_{b} \mid a_{\mathbf{k}}^{\lambda}\right)=0$.

In a classical fluid there are $(d-1)$ diffusive shear modes $\left(\lambda=\eta_{i}: i=1,2, \ldots, d-1\right)$, being the components of the tranversal flow field $\mathbf{u}_{\mathbf{k} \perp}=\mathbf{u}_{\mathbf{k}}-\hat{\mathbf{k}} \hat{\mathbf{k}} \cdot \mathbf{u}_{\mathbf{k}}$, with decay rates $z_{k}^{\eta}=-\nu k^{2}$, and shear viscosity $\eta=\rho \nu$, and one diffusive heat mode $a_{k}^{H}$ with decay rate $z_{\mathbf{k}}^{H}=-D_{T} k^{2}$ and heat diffusivity $D_{T}$. In addition there are two damped propagating sound modes $(\lambda=\sigma= \pm)$ with decay rate $z_{k}^{\sigma}=-i \sigma c_{0} k-\frac{1}{2} \Gamma_{s} k^{2}$, where $c_{0}$ is the speed of sound and $\Gamma_{s}$ the sound damping constant. For the explicit expressions of fluid modes $a_{\mathbf{k}}^{\lambda}$ in terms of conserved densities, and the calculation of the dominant LTT's we refer to [2].

A special case is the single-particle TCF's $\left\langle j_{b}(t) j_{b}(0)\right\rangle$ in fluids. Here the mode coupling formula necessarily involves the Fourier mode of the tagged particle density, i.e., the selfdiffusion mode, $a_{\mathbf{k}}^{s}=n_{\mathbf{k}}^{s}=\exp \left[-i \mathbf{k} \cdot \mathbf{r}_{1}\right]$, where $i=1$ is the label of the tagged particle. Consequently, the following replacements have to be made in (3): $\mu \rightarrow s$ and $\frac{1}{2} \Sigma_{\lambda \mu} \rightarrow \Sigma_{\lambda}$. Moreover, if one of the labels equals $s$, then the amplitude $A_{b}^{\lambda s}$ $=\left(j_{b} \mid a_{\mathbf{k}}^{\lambda} a_{-\mathbf{k}}^{s}\right) \equiv\left\langle j_{b} a_{\mathbf{k}}^{\lambda} a_{-\mathbf{k}}^{s}\right\rangle$ without a factor $1 / V$. To extract the long-time behavior from (3) we change the integration variable $\mathbf{k}$ to $\mathbf{q} / \sqrt{t}$, and take the long-time limit, using $a_{\mathbf{k}}^{\lambda} \rightarrow a_{\mathbf{0}}^{\lambda}$ and $n_{\mathbf{k}}^{s} \rightarrow n_{\mathbf{0}}^{s}=1$. So, the amplitude of the single particle function simplifies to $A_{b}^{\lambda s}=\left\langle j_{b} \mid a_{0}^{\lambda}\right\rangle$, and depends only on the direction $\hat{\mathbf{k}}$ of the wave vector $\mathbf{k}$. The final result for the LTT becomes

$$
\left\langle j_{b}(t) j_{b}(0)\right\rangle \simeq \sum_{\lambda} \overline{\left[\left\langle j_{b} \mid a_{0}^{\lambda}\right\rangle\right]^{2}} /\left[4 \pi\left(D_{\lambda}+D\right) t\right]^{d / 2},
$$

where only the diffusive modes $\left[\lambda=\left(\eta_{i}, H\right)\right]$ contribute. The overline indicates an average over the solid angle $\hat{\mathbf{k}}$. As the calculations of $A_{b}^{\lambda s}$ are similar to those in Ref. [2(b)], we simply quote the final results for the LTT in the equilibrium TCF's of classical fluids, $C_{b}(t)=\left\langle j_{b}(t) j_{b}(0)\right\rangle /\left\langle j_{b}^{2}\right\rangle$ with $b$ $=\{1,2,3,4, \ldots\}$, and $j_{b}=\left(v_{x}, v^{2}-\left\langle v^{2}\right\rangle, v^{2} v_{x}, v^{4}-\left\langle v^{4}\right\rangle\right)$, i.e.,

$$
\begin{gathered}
C_{1}(t) \simeq[(d-1) / n d][4 \pi(\nu+D) t]^{-d / 2}, \\
C_{2}(t) \simeq\left(\mathcal{C}_{v}^{0} / n \mathcal{C}_{p}\right)\left[4 \pi\left(D_{T}+D\right) t\right]^{-d / 2},
\end{gathered}
$$

with $\mathcal{C}_{p}$ the specific heat at constant pressure, and $\mathcal{C}_{v}^{0}=\frac{1}{2} d k_{B}$. One similarly shows that odd-in- $v$, or even-in- $v$ correlations are at large times proportional to $C_{1}(t)$ and $C_{2}(t)$, i.e., $C_{3}(t)$ $\simeq[(d+2) /(d+4)] C_{1}(t), \quad C_{4}(t) \simeq[(d+2) /(d+3)] C_{2}(t), \quad$ etc. for $k=5,6, \ldots$. The result for the VACF in hard sphere or Lennard-Jones fluids is well known [1,2]. The above results also disprove the misconception that momentum conservation is necessary for the existence of LTT's in classical fluids. The results in Eqs. (4) and (5) for classical fluids also apply to DPD fluids with energy and momentum conservation [8]. In DPD fluids without energy conservation [7] only the relations for odd- $k$ values apply because the heat mode is absent, and the system is thermostated instantaneously.

Next we want to extend these results to the random DPD solid. The mode coupling results (3) and (4) for TCF's in fluids do not, in general, apply to systems with quenched disorder [12]. There they apply only to TCF's $C(t \mid X)$, calculated in a nonuniform equilibrium state, corresponding to a single quenched configuration $X$, where $A^{\lambda \mu}(X)$ and $D_{T}(X)$ in (3) and (4) depend on the configuration $X$. To obtain the full correlation, $C(t)=\langle C(t \mid X)\rangle$, a subsequent average over all quenched configurations has to be performed. In general, the $X$ dependence of $A^{\lambda \mu}(X)$ and $D_{T}(X)$ is not known explicitly. A more phenomenological derivation of a mode coupling formula for diffusive systems with quenched disorder has been presented in Ref. [12], and one can extend that method to the heat conducting random DPD solid.

First, we investigate only those special cases for which the LTT of $C(t \mid X)$ can be determined explicitly from Eq. (3). Consider the single particle energy correlation $C_{e}(t \mid X)$ $=\left\langle\delta \epsilon_{i}(t) \delta \epsilon_{i}(0)\right\rangle_{X}$ in the DPD solid, where the heat mode, $a_{\mathbf{k}}^{H}(t)=a_{\mathbf{k}}^{H}(0) \exp \left[-t k^{2} D_{T}(X)\right]$, is the only slow macroscopic mode, and $a_{\mathbf{k}}^{H}=e_{\mathbf{k}} / \sqrt{\left(e_{\mathbf{k}} \mid e_{\mathbf{k}}\right)}$. In the long-time limit (where $\mathbf{k} \rightarrow 0)$ the relevant amplitude $A^{H s}(X)=\left\langle\delta \epsilon_{1} a_{\mathbf{k}}^{H} a_{-\mathbf{k}}^{s}\right\rangle_{X}$ $\simeq\left\langle\delta \epsilon_{1} a_{0}^{H}\right\rangle_{X}=\sqrt{V\left\langle(\delta \epsilon)^{2}\right\rangle / N}$ where $a_{\mathbf{k}}^{s}=\exp \left[-i \mathbf{k} \cdot \mathbf{r}_{1}\right]$ is a frozen mode with $z_{k}^{s}=0$. Inserting these results in (4), and performing the $X$ average yields the LTT

$$
C_{e}(t) \simeq \frac{V}{N}\left(\frac{1}{4 \pi D_{T} t}\right)^{d / 2}=\frac{1}{\rho\left[4 \pi t^{*}\right]^{d / 2}} .
$$

Here $\left\langle D_{T}(X)\right\rangle=D_{T}$, and fluctuation corrections of relative order $\left\langle\left(\delta D_{T}(X)\right)^{2}\right\rangle / D_{T}$ have been neglected. Moreover, $t^{*}$ $=D t / r_{c}^{2}$ and $\rho=N r_{c}^{2} / V$ is proportional to the mean number of particles inside an interaction sphere of radius $r_{c}$. If the particles are put on a lattice (no disorder), the LTT in $C_{e}(t)$ applies as well to the lattice version of the DPD solid. Both on-lattice and off-lattice computer simulations of the LTT in $C_{e}$ are in excellent agreement [11] with the theoretical prediction (6). 
Next we study the $N$-particle correlation $C_{Q}(t)$ $=\left\langle Q_{x}(t) Q_{x}(0)\right\rangle / V$. The random DPD solid sustains a microscopic heat flux, $Q_{x}=Q_{D}+Q_{R}$, with a dissipative $(D)$ and a random part $(R)$, as given explicitly in [10]. We start with $Q_{D}(t)=\kappa_{0} \Sigma_{i<j} w\left(r_{i j}\right) r_{i j, x}\left[\epsilon_{j}(t)-\epsilon_{i}(t)\right]$. This sum of dissipative pair interactions represents the instantaneous exchange of energy over a distance $r_{i j}$ through the interactions (collisional transfer). The model parameter $\kappa_{0}$ represents the interaction frequency, and the range function is a step function, vanishing for $r>r_{c}$, and normalized such that $\int d \mathbf{r} w(r)=r_{c}^{d}$. Consequently $\kappa_{0}\left(\epsilon_{j}-\epsilon_{i}\right) w\left(r_{i j}\right)$ is the rate of energy transfer between the interacting pair $(i j)$ with $r_{i j}<r_{c}$. The corresponding TCF is $C_{D}(t)=V^{-1}\left\langle Q_{D}(t) Q_{D}(0)\right\rangle$.

The next object to be studied is the stochastic part of the heat flux $Q_{R}$, and the corresponding correlation $C_{R}(t)$ $=V^{-1}\left\langle Q_{R}(t) Q_{R}(0)\right\rangle$ and cross correlations $C_{D R}(t)$ and $C_{R D}(t)$. For our present purpose it is sufficient to note [10] that $Q_{R}$ is a linear combination of the Langevin forces, $\widetilde{F}_{i j}(t)=-\widetilde{F}_{j i}(t)$, with coefficients depending on $\epsilon_{i}$ and $\epsilon_{j}$ (multiplicative noise), where $\left\langle\widetilde{F}_{i j}(t)\right\rangle=0$ and $\left\langle\widetilde{F}_{i j}(t) \widetilde{F}_{k l}\left(t^{\prime}\right)\right\rangle=\delta\left(t-t^{\prime}\right)\left(\delta_{i k} \delta_{j l}\right.$ $\left.-\delta_{i l} \delta_{j k}\right)$. The latter property guarantees that $C_{R}(t)$ is deltacorrelated in time, $C_{R}(t) \simeq\left(2 \lambda_{\infty} / k_{B} \beta^{2}\right) \delta(t)$. Here $k_{B}$ is Boltzmann's constant, $\beta=1 / k_{b} T$ the inverse temperature, and $\lambda_{\infty}$ the heat conductivity in mean-field approximation $(\rho \rightarrow \infty)$, as calculated in [10].

The cross correlations, $C_{D R}$ and $C_{R D}$ vanish, being linear in $\widetilde{F}_{i j}$. The heat conducting DPD solid is, in fact, a diffusive system with the static disorder [12] with heat conduction $\lambda$ $=k_{B} \beta^{2} \int_{0}^{\infty} d t C_{Q}(t)$, and we apply the mode coupling theory developed for such systems in Ref. [12] to calculate the LTT of $C_{Q}(t)$. To do so the local concentration, $c(r, t)$ in the fluctuating diffusion equation of Ref. [12] needs to be replaced by the energy density, $e(r, t)$, to find

$$
\partial e(r, t) / \partial t=\nabla \cdot \lambda(r, X) \cdot \nabla[e(\mathbf{r}, t) / \psi(\mathbf{r}, X)],
$$

where $\psi(\mathbf{r}, X)=C n(\mathbf{r})$ and $C$ is the specific heat per DPD particle. The tensor $\lambda^{\alpha \beta}(\mathbf{r}, X)$ is the spatially fluctuating heat conductivity tensor with $\left\langle\lambda^{\alpha \beta}(\mathbf{r}, X)\right\rangle=\lambda \delta_{\alpha \beta}$, and the heat diffusivity is $D_{T}=\lambda /\langle\psi\rangle=\lambda / C n$. Following the derivation of Ref. [12] one finds for the LTT,

$$
C_{Q}(t) \simeq C_{D}(t) \simeq-\pi n k_{B} T^{2} C \Delta /\left(4 \pi D_{T} t\right)^{1+d / 2},
$$

where $\Delta$ is the mean square fluctuation in the $\mathbf{k}=0$ Fourier components of $\delta D^{\alpha \beta}(\mathbf{r}, X)=\delta \lambda^{\alpha \beta}(\mathbf{r}, X) /[C n]$, i.e.,

$$
\Delta=(1 / d V)\left\langle\delta D_{0}^{\alpha \beta}(\mathbf{r}, X) \delta D_{0}^{\beta \alpha}(\mathbf{r}, X)\right\rangle
$$

with implied summation convention for repeated indices.

The LTT $\sim t^{-1-d / 2}$ of $C_{D}$ in the random solid has the same sturcture as the VACF in the Lorentz gas, and both LTT's vanish when the fixed particles are filling the sites of a periodic lattice [13], because $\Delta=0$.
In the present system one has for large densities the explicit expression for the fluctuating heat diffusivity

$$
D^{\alpha \beta}(\mathbf{r}, X)=\left(\kappa_{0} / n V\right) \sum_{1<j} w\left(r_{i j}\right) r_{i j, \alpha} r_{i j, \beta},
$$

and the quantity $\Delta$ can be calculated, yielding the LTT at large densities,

$$
\frac{C_{D}(t)}{C_{D}(0)} \simeq \frac{\pi}{\rho}\left(\frac{\mathrm{d}+2}{\mathrm{~d}+4}\right)\left(\frac{1}{4 \pi t^{*}}\right)^{1+d / 2} .
$$

Do the above results have any implications for dense classical fluids, say in the vicinity of the triple point? We propose the following scenario. In such dense systems the motion of the particles is quite restricted-somewhat comparable to a quenched system - and the collisional transfer of momentum and energy is the dominant transport mechanism. Consider for example the microscopic stress tensor. If the rate of energy transfer between the particle pair $(i j)$ in $Q_{D}$ is replaced by the rate of momentum transfer, i.e., the interparticle force, $F_{i j, y}=-\partial V\left(r_{i j}\right) / \partial r_{i j, y}$, then $Q_{x}$ becomes the collisional transfer component $S_{x y}^{c}$ of the stress tensor. This suggests that in dense fluids the stress correlation function has a LTT, $C_{S}^{c}(t)$ $=V^{-1}\left\langle S_{x y}^{c}(t) S_{x y}^{c}(0)\right\rangle \sim A_{c} t^{-1-d / 2}$, similar to the velocity autocorrelation function in the Lorentz gas [12]. Standard mode coupling theory for fluids only predicts that the TCF $C_{S}^{k}(t)$ of the kinetic stresses, $S_{x y}^{k}=\sum_{i} m v_{i x} v_{i y}$, has a LTT $\sim A_{k} t^{-d / 2}[2,5,14]$, and that no such tail is present in $C_{S}^{c}(t)$.

Standard molecular dynamics (MD) simulations of TCF's in hard sphere fluids [14] have not been able to establish a LTT $\sim t^{-d / 2}$ in $C_{S}^{c}$, as is consistent with theory. However, the nonequilibrium MD simulations [15] for small LennardJones systems under a constant shear rate $\dot{\gamma}$ seem to suggest a (possibly intermediate) LTT of $C_{S}^{c}(t) \sim A_{c} t^{-d / 2}$ with $A_{c}$ one or two orders of magnitude larger than the value $A_{k}$, predicted by the theory for $C_{S}^{k}$. Here determination of the LTT involves two nonuniform limits $(\dot{\gamma} \rightarrow 0, t \rightarrow \infty)$. A large intermediate tail $\sim t^{-d / 2}$ may be a crossover phenomenon for $t$ $<t_{\text {cross }}(\dot{\gamma})$ at a small, but nonvanishing $\dot{\gamma}$, where the collisional transfer stress correlation in dense fluids is showing the $t^{-d / 2}$-tail of Burnett-type correlations, similar to those derived for Lorentz gases [12].

It would be of great interest to test the proposed scenario for LTT's in TCF's in dense classical fluids by performing detailed computer simulations of $C_{Q}$ in DPD solids and fluids, as well as by developing a quantitative analysis of $C_{Q}$ for general densities. Promising alternatives of measuring these LTT's, particularly in one dimension, are offered by simulating the Helfand moments [16].

The author acknowledges stimulating discussions and/or correspondence with E. Ben-Naim, J. Machta, J.R. Dorfman, H. van Beijeren, M. Ripoll, and I. Pagonabarraga. 
[1] T. Wainwright, B. J. Alder, and D. M. Gass, Phys. Rev. A 4, 233 (1971); D. Levesque and W. Ashurst, Phys. Rev. Lett. 33, 277 (1974).

[2] (a) L. P. Kadanoff and J. Swift, Phys. Rev. A 136, 1519 (1964); (b) M. H. Ernst, E. H. Hauge, and J. M. J. van Leeuwen, J. Stat. Phys. 15, 7 (1976).

[3] R. J. Glauber, J. Math. Phys. 4, 294 (1963).

[4] Lattice Gas Methods for Partial Differential Equations, edited by G. D. Doolen (Addison-Wesley, Reading, MA, 1990).

[5] D. Frenkel and I. Pagonabarraga (private communication).

[6] E. Ben-Naim and P. Krapivsky, in Granular Gas Dynamics, edited by T. Pöschel and N. Brilliantov (Springer Verlag, Berlin, 2003) p. 65; E. Ben-Naim (private communication).

[7] P. Español and P. Warren, Europhys. Lett. 30, 191 (1995); C. A. Marsh, G. Backx, and M. H. Ernst, Phys. Rev. E 56, 1676 (1997); M. Ripoll, M. H. Ernst, and P. Español, J. Chem. Phys. 15, 7271 (2001).

[8] J. Bonet-Avalós and A. D. Mackie, Europhys. Lett. 40, 141 (1997); P. Español, ibid. 40, 631 (1997).
[9] M. H. J. Hagen, C. P. Lowe, and D. Frenkel, Phys. Rev. E 51, 4287 (1995).

[10] M. Ripoll and M. H. Ernst, e-print cond-mat/0406026, Phys. Rev. E (to be published).

[11] M. Ripoll and M. H. Ernst, LANL Report No. LA-UR-046774. 2004 (unpublished).

[12] M. H. Ernst, J. Machta, J. R. Dorfman, and H. van Beijeren, J. Stat. Phys. 34, 477 (1984); 35, 413 (1985).

[13] A. K. Harrison, and R. Zwanzig, J. Stat. Phys. 42, 935 (1986).

[14] A. J. C. Ladd, W. E. Alley, and B. J. Alder, J. Stat. Phys. 48, 1147 (1987); A. J. C. Ladd and B. J. Alder, ibid. 57, 473 (1989).

[15] D. J. Evans, J. Stat. Phys. 22, 81 (1980); Phys. Rev. A 23, 1988 (1981); G. Marcelli, B. D. Todd, and R. J. Sadus, Phys. Rev. E 63, 021204 (2001).

[16] M. P. Allen and D. J. Tildesley, Computer Simulations in Liquids (Clarendon, Oxford, 1987); S. Hess, M. Kröger, and D. Evans, Phys. Rev. E 67, 042201 (2003). 